Pacific Journal of Mathematics

BOUNDARY VALUE PROBLEMS FOR NONLINEAR ORDINARY 


\title{
BOUNDARY VALUE PROBLEMS FOR NONLINEAR ORDINARY DIFFERENTIAL EQUATIONS
}

\author{
H. A. Antosiewicz
}

\begin{abstract}
Conditions are given under which a quasi-linear differential equation has at least one solution in a given compact interval that satisfies a given system of homogeneous or nonhomogeneous linear constraints. These conditions are not formulated in the space in which the solutions take their values, as is usually done; instead they involve the set of continuous mappings subject to the constraints and the set of forcing terms for which the associated nonhomogeneous linear differential equation has solutions satisfying the constraints. The latter set is, under mild conditions, a topological direct summand of the space of continuous mappings. This occurs in the problem of the existence of periodic solutions which is discussed in detail as illustration.
\end{abstract}

In the present note we derive simple sufficient conditions in order that a differential equation

$$
x^{\prime}=A(t) x+f(t, x)
$$

have at least one solution $u$ in a compact interval $K$ which satisfies a system of constraints of the form

$$
c_{i}(u)=\eta_{i}, \quad 1 \leqq i \leqq m .
$$

Here $\left(c_{i}\right)$ is a linearly independent family of continuous linear forms on the Banach space $C$ of continuous mappings of $K$ into $X$, the underlying real Banach space, and $y=\left(\eta_{i}\right)$ is an arbitrary point in $R^{m}$.

Our results are in the spirit of two very general theorems, essentially due to Corduneanu [7], which Hartman and Onuchic [9] have applied to the asymptotic integration of differential equations such as (1). However, unlike these theorems, our considerations do not depend upon the work of Massera and Schäffer (see, e.g., [11], [12]) but instead are based on some elementary facts concerning linear differential equations that admit solutions for which (2) holds. An entirely different treatment of boundary value problems has recently been given by Conti [4], [5], [6].

For the sake of simplicity, we assume throughout that $t \rightarrow A(t)$ is a continuous mapping of $K$ into the normed space of continuous endomorphisms of $X$ and that $f$ is a continuous mapping of $K \times K$

Received January 6, 1965. This work was done with partial support from the National Science Foundation and the Army Research Office (DURHAM). 
into $X$. Much less restrictive assumptions would suffice.

2. Let $\Gamma$ be the mapping $c \rightarrow\left(c_{i}(c)\right)_{1 \leqq i \leqq m}$ of $C$ into $R^{m}$ and denote by $V$ the inverse image by $\Gamma$ of any $y \in R^{m}$. Since $\Gamma$ is a continuous linear surjection, $\boldsymbol{V}$ is a closed linear variety of codimension $m$ in $\boldsymbol{C}$.

We first examine under what conditions a linear differential equation

$$
x^{\prime}=A(t) x+b(t)
$$

with $b \in \boldsymbol{C}$ has at least one solution which belongs to $\boldsymbol{V}$.

Corresponding to some (fixed) $t_{0} \in K$, define $\phi: C \rightarrow C$ to be the mapping which associates with each $b \in C$ the particular solution of (3) that equals $0 \in X$ at $t_{0}$, and let $\psi: X \rightarrow C$ be the mapping whose value at each $x \in X$ is the solution of the homogeneous equation associated with (3) which equals $x$ at $t_{0}$. Clearly, $\phi$ and $\psi$ are continuous linear injections, and every solution $v$ of (3) in $K$ has the unique representation $v=\psi(x)+\phi(b)$ where $x=v\left(t_{0}\right)$. It follows that (3) has a solution in $\boldsymbol{V}$ if and only if the equation

$$
\Gamma \circ \psi(x)=y-\Gamma \circ \phi(b)
$$

has a solution in $X$.

Set $Y=\Gamma \circ \psi(X), \Phi=-\Gamma \circ \phi$, and define $\boldsymbol{B}$ as the inverse image by $\Phi$ of the closed linear variety $-y+Y$ in $R^{m}$. Then $B$ is either empty or a closed linear variety in $\boldsymbol{C}$. Indeed, in the latter case, every equation (3) with $b \in \boldsymbol{B}$ has at least one solution belonging to $\boldsymbol{V}$.

Observe that the null space $X_{0}$ of $\Gamma \circ \psi$ is a closed linear subspace of $X$ whose codimension is at most equal to $m$. Hence $X_{0}$ admits a topological supplement $X_{1}$, and there exists a continuous endomorphism $P$ of $X$ which projects $X$ along $X_{1}$ onto $X_{0}$. Moreover, since the restriction of $\Gamma \circ \psi$ to $X_{1}$ is obviously an isomorphism of $X_{1}$ onto $Y$, there is a constant $\lambda>0$ and, for each $z \in Y$, a unique $x_{1} \in X_{1}$ such that $\Gamma \circ \psi\left(x_{1}\right)=z$ and $\left\|x_{1}\right\| \leqq \lambda\|z\|$.

LEMMA 1. If $\boldsymbol{B}$ is nonempty, there exist positive constants $\alpha, \beta$, $\gamma$ such that, given any $x_{0} \in X_{0}$, (3) has for every $b \in \boldsymbol{B} a$ unique solution $v \in \boldsymbol{V}$ with $P v\left(t_{0}\right)=x_{0}$ for which

$$
\|v\| \leqq \alpha\left\|x_{0}\right\|+\beta\|y\|+\gamma\|b\| \text {. }
$$

The proof is an immediate consequence of our preceding remarks.

Lemma 1 shows that, if $\boldsymbol{B}$ is nonempty, (3) induces a mapping $\sigma:\left(x_{0}, b\right) \rightarrow v$ of $X_{0} \times \boldsymbol{B}$ into $\boldsymbol{V}$ which is continuous. For, whatever $\left(x_{0}, b_{1}\right),\left(\widehat{x}_{0}, b_{2}\right)$ in $X_{0} \times \boldsymbol{B}$, the mapping $w=\sigma\left(x_{0}, b_{1}\right)-\sigma\left(\hat{x}_{0}, b_{2}\right)$ is a solution of (3) with $b=b_{1}-b_{2}$ such that $P w\left(t_{0}\right)=x_{0}-\widehat{x}_{0}$ and $\Gamma(w)=0$, 
and this implies by (5)

$$
\left\|\sigma\left(x_{0}, b_{1}\right)-\sigma\left(\widehat{x}_{0}, b_{2}\right)\right\| \leqq \alpha\left\|x_{0}-\widehat{x}_{0}\right\|+\gamma\left\|b_{1}-b_{2}\right\| \text {. }
$$

It may happen that $y \in Y$ or, equivalently, that $\boldsymbol{B}$ is a closed linear subspace of $C$. This occurs if and only if the homogeneous equation associated with (3) has at least one solution which belongs to $\boldsymbol{V}$. Of course, this is always the case when $y=0$.

Lemma 2. If $y \in Y$ and $\Gamma \circ \phi \circ \psi$ is surjective, then $\boldsymbol{B}$ is a topological direct summand of $\boldsymbol{C}$.

Proof. Since $\Gamma \circ \phi \circ \psi$ is a continuous linear mapping of $X$ onto $R^{m}$ whose null space is a topological direct summand of $X$, there exists a continuous linear injection $M: R^{m} \rightarrow X$ which is the right inverse of $\Gamma \circ \phi \circ \psi$. Define the injection $\Psi=-\psi \circ M$ of $R^{m}$ into $C$ and let $Q$ be the projection of $R^{m}$ along $Y$ onto any supplement of $Y$. We assert that $\tau=\Psi \circ Q \circ \Phi$ is a continuous projection of $\boldsymbol{C}$ for which $\tau^{-1}(0)=\boldsymbol{B}$. Clearly, $\tau$ is a continuous endomorphism of $\boldsymbol{C}$. It is idempotent, hence a projection, because $\Phi \circ \Psi$ is the identity mapping of $R^{m}$. Moreover, $\tau(c)=0$ for some $c \in C$ is equivalent to $Q \circ \Phi(c)=0$ which, in turn, is equivalent to $c \in \Phi^{-1}(Y)$. Since $y \in Y$ implies $\boldsymbol{B}=\Phi^{-1}(Y)$, it follows that $\tau^{-1}(0)=\boldsymbol{B}$.

3. We now turn our attention to the nonlinear differential equation (1).

For convenience we introduce, for every $b \in V$, the injection $g_{b}: t \rightarrow(t, b(t))$ of $K$ into $K \times X$ so that we may write $f \circ g_{b}$ for the continuous mapping $t \rightarrow f(t, b(t))$ of $K$ into $X$. The constants $\alpha, \beta, \gamma$ will always be those referred to in Lemma 1 .

Evidently, a necessary condition for (1) to have a solution in $K$ belonging to $\boldsymbol{V}$ is that $\boldsymbol{B}$ be nonempty. Therefore we will make this assumption in the sequel, without further mention. To obtain sufficient conditions we proceed in the following way, which suggests itself rather naturally.

Suppose there is a closed ball $B$ in $V$ such that $f \circ g_{b} \in \boldsymbol{B}$ for every $b \in B$. Then (1) gives rise to the mapping $\Sigma:\left(x_{0}, b\right) \rightarrow \sigma\left(x_{0}, f \circ g_{b}\right)$ of $X_{0} \times B$ into $V$; of course, $\Sigma\left(x_{0}, b\right)$ is the unique solution $v$ of the linear differential equation

$$
x^{\prime}=A(t) x+f(t, b(t))
$$

which belongs to $V$ and satisfies $P v\left(t_{0}\right)=x_{0}$. It is easy to see that $\Sigma$ is continuous. For the continuity of $f$ in $K \times X$ implies that the mapping $b \rightarrow f \circ g_{b}$ of $B$ into $B$ is continuous, and the continuity of $\sigma: X_{0} \times \boldsymbol{B} \rightarrow \boldsymbol{V}$ was shown previously. Clearly, every $u \in B$ such that 
$\Sigma\left(x_{0}, u\right)=u$ for some $x_{0} \in X_{0}$ is a solution of (1) in $V$ with $P u\left(t_{0}\right)=x_{0}$. Thus, we only need to look for further conditions under which the mapping $b \rightarrow \Sigma\left(x_{0}, b\right)$ of $B$ into $V$ has a fixed point for some $x_{0} \in X_{0}$.

THEOREM 1. Let $a \geqq 0, r>0, \sigma>0$ be constants such that $\alpha a+\beta\|y\|+\gamma \rho \leqq r$ and denote by $H_{0}$ the closed ball in $X_{0}$ with center at 0 and radius $a$. If $X$ has finite dimension and if $b \in \boldsymbol{V}$ and $\|b\| \leqq r$ implies $f \circ g_{b} \in \boldsymbol{B}$ and $\left\|f \circ g_{b}\right\| \leqq \rho$, then (1) admits, for each $x_{0} \in H_{0}$, at least one solution $u \in V$ with $\|u\| \leqq r$ for which $P u\left(t_{0}\right)=x_{0}$.

Proof. The ball $B=\{b \in V:\|b\| \leqq r\}$ is a convex closed subset of $C$, because $V$ is closed. Lemma 1 and our assumptions show that $\Sigma\left(x_{0}, B\right) \subset B$ for any $x_{0} \in H_{0}$. From this we deduce at once that the set $\left\{v(t): v \in \Sigma\left(x_{0}, B\right)\right\}$, for each $t \in K$, is relatively compact in $X$. Moreover, since any $v \in \Sigma\left(x_{0}, B\right)$ is a solution of (7) for some $b \in B$ and so satisfies, for every $t, s$ in $K$,

$$
\|v(t)-v(s)\| \leqq r\left|\int_{s}^{t}\|A(\tau)\| d \tau\right|+\rho|t-s|,
$$

$\Sigma\left(x_{0}, B\right)$ is equicontinuous and therefore, by Ascoli's theorem, relatively compact in $C$. Thus, Schauder's theorem implies, for each $x_{0} \in H_{0}$, the existence of at least one $u \in B$ such that $\Sigma\left(x_{0}, u\right)=u$.

THEOREM 2. Let $a \geqq 0$ and positive constants $k, r, \rho$ be so chosen that $\alpha a+\beta\|y\|+\gamma \rho \leqq r,(k \gamma<1), 2 k r<\rho$, and denote by $H$ the closed ball in $X$ with center at 0 and radius $r$. Suppose

(i) $f$ is Lipschitzian in $K \times H$ for the constant $k$;

(ii) $f \circ g_{b} \in \boldsymbol{B}$ for every $b \in \boldsymbol{V}$ with $\|b\| \leqq r$;

(iii) there is a $b_{0} \in V$ with $\left\|b_{0}\right\| \leqq r$ such that $\left\|f \circ g_{b_{0}}\right\| \leqq \rho-2 k r$. Then (1) admits, for each $x_{0} \in H_{0}$, a unique solution $u_{x_{0}} \in V$ with $\left\|u_{x_{0}}\right\| \leqq r$ for which $P u_{x_{0}}\left(t_{0}\right)=x_{0}$. Moreover, the mapping $x_{0} \rightarrow u_{x_{0}}$. is continuous in $H_{0}$.

Proof. The ball $B=\{b \in V:\|b\| \leqq r\}$ is a complete subspace of $C$. Since, by our assumptions, $\left\|f \circ g_{b}\right\| \leqq \rho$ for every $b \in B$, Lemma 1 permits us to define a sequence $\left(v_{n}\right)$ of points in $B$ such that $v_{0}=b_{0}$ and $v_{n}=\Sigma\left(x_{0}, v_{n-1}\right)$ for $n \geqq 1$. By induction we may write $v_{n}=u_{n}\left(x_{0}\right)$ where each $u_{n}: H_{0} \rightarrow B$ is continuous. From (6) we infer then that

$$
\left\|u_{n}\left(x_{0}\right)-u_{n-1}\left(x_{0}\right)\right\| \leqq k \gamma\left\|u_{n-1}\left(x_{0}\right)-u_{n-2}\left(x_{0}\right)\right\| \leqq 2 r(k \gamma)^{n-1}
$$

for any $\dot{x}_{0} \in H_{0}$, and this implies that $\left(u_{n}\right)$ converges uniformly in $H_{0}$. Thus $u_{x_{0}}=\lim u_{n}\left(x_{0}\right)$ exists for every $x_{0} \in H_{0}$, and $x_{0} \rightarrow u_{x_{0}}$ is continuous in $H_{0}$. It immediately follows that $u_{x_{0}} \in B$ and $\Sigma\left(x_{0}, u_{x_{0}}\right)=u_{x_{0}}$ for every 
$x_{0} \in H_{0}$. Clearly, $u_{x_{0}}$ is unique. If $w$ would be another solution of (1) in $V$, satisfying $\|w\| \leqq r$ and $P w\left(t_{0}\right)=x_{0}$, then $v=w-u_{x_{0}}$ is a solution of the linear equation (3) with $b=f \circ g_{w}-f \circ g_{u_{x_{0}}}$ such that $P v\left(t_{0}\right)=0$ and $\Gamma(v)=0$. Since, by Lemma 1 , there is only one such solution, we conclude that $v=\sigma(0, b)$, and this implies by (6)

$$
\left\|w-u_{x_{0}}\right\| \leqq \gamma\left\|f \circ g_{w}-f \circ g_{u_{x_{0}}}\right\| \leqq k \gamma\left\|w-u_{x_{0}}\right\| \text {. }
$$

Hence $w=u_{x_{0}}$ because $k \gamma<1$, which completes the proof.

These theorems are, in a sense, concrete versions adapted to our setting, of the two very general theorems in [9] which are used there for a completely different purpose.

We can say more when $y \in Y$ and $\boldsymbol{B}$ is a topological direct summand of $C$. For in this case, there exists a continuous projection $\tau$ of $C$ with $\tau^{-1}(0)=\boldsymbol{B}$ and, for every $b \in \boldsymbol{C}$, and unique $h_{b} \in \boldsymbol{C}$ such that $f \circ g_{b}-h_{b} \in \boldsymbol{B}$. Obviously, $h_{b}=\tau\left(f \circ g_{b}\right)$ so that $\left\|h_{b}\right\| \leqq\|\tau\|\left\|f \circ g_{b}\right\|$ and $\left\|f \circ g_{b}-h_{b}\right\| \leqq 2\|\tau\|\left\|f \circ g_{b}\right\|$. Thus, even if $f \circ g_{b}$ does not belong to $\boldsymbol{B}$ for any $b \in \boldsymbol{V}$, the linear equation

$$
x^{\prime}=A(t) x+f(t, b(t))-h_{b}(t)
$$

still admits, for each $x_{0} \in X_{0}$, a unique solution $v \in V$ for which $P v\left(t_{0}\right)=x_{0}$. This yields immediately the following result.

Corollary 1. Suppose $X$ has finite dimension, $y \in Y$ and $\boldsymbol{B}$ is a topological direct summand of $\boldsymbol{C}$. Let $\tau$ be a continuous projection of $\boldsymbol{C}$ with $\tau^{-1}(0)=\boldsymbol{B}$ and let $a \geqq 0, r>0, \rho>0$ be constants such that $\alpha a+\beta\|y\|+2 \gamma \rho\|\tau\| \leqq r . \quad$ If $\left\|f \circ g_{b}\right\| \leqq \rho$ for every $b \in V$ with $\|b\| \leqq r$, then there exists, for each $x_{0} \in H_{0}$, at least one differentiable mapping $u \in V$ with $\|u\| \leqq r$ for which $P u\left(t_{0}\right)=x_{0}$ and

$$
u^{\prime}(t)=A(t) u(t)+f(t, u(t))-h_{u}(t)
$$

at every $t \in K$.

It follows that $u$ will be a solution of (1) in $V$ if and only if $h_{u}=0$. Of course, $h_{u}(t)=0$ is the familiar bifurcation equation.

Theorem 2 admits a similar corollary; we leave its precise formulation to the reader because we will state a special case of it presently.

4. We conclude by illustrating our foregoing considerations on an important example which we discussed in [1] without giving details. It has been treated by various other methods of different generality (see, e.g., [2]-[6], [8], [10]).

Suppose $X$ is the real normed space $R^{n}, K$ the compact interval $[0, T]$, and let us inquire into the existence of a "periodic" solution $u$ of (1) in $K$ such that $u(0)=u(T)$. 
Here $\Gamma$ is simply the mapping $c \rightarrow c(0)-c(T)$ of $C$ into $R^{n}$ and hence $\boldsymbol{V}$ is the Banach space of "periodic" mappings $c \in \boldsymbol{C}$ for which $c(0)-c(T)=0$. If we choose $t_{0}=0$ and denote by $U$ the particular solution of the homogeneous matrix equation associated with (3), which equals the identity matrix $I$ at $t=0$, then we may write the mappings $\psi: R^{n} \rightarrow C$ and $\phi: C \rightarrow C$ explicitly in the form

$$
\psi(x): t \rightarrow U(t) x, \quad \phi(b): t \rightarrow U(t) \int_{0}^{t} U^{-1}(s) b(s) d s .
$$

Thus, $X_{0}$ is the null space, and $Y$ the range, of the mapping $I-U(T)$. The mapping $\Gamma \circ \phi \circ \psi$ is immediately computed to be the bijection $x \rightarrow-T U(T) x$, and so the assumptions of Lemma 2 are satisfied.

The set $\boldsymbol{B}$ of mappings $b \in \boldsymbol{C}$ for which the linear differential equation (3) has at least one "periodic" solution $v \in \boldsymbol{V}$ is a topological direct summand of $C$.

A topological supplement to $\boldsymbol{B}$ may be constructed by choosing $Q$ (in the proof of Lemma 2), for example, as the projection of $R^{n}$ onto the null space $Y_{0}$ of the adjoint of $I-U(T)$, which is a (orthogonal) supplement to the range of $I-U(T)$. Then $\boldsymbol{B}$ is the null space of the continuous projection $\tau=\Psi \circ Q \circ \Phi$ where $\Phi: C \rightarrow R^{n}$ is the mapping

$$
b \rightarrow U(T) \int_{0}^{T} U^{-1}(s) b(s) d s
$$

and $\Psi: R^{n} \rightarrow C$ the mapping such that

$$
\Psi(x): t \rightarrow \frac{1}{T} U(t) U^{-1}(T) x .
$$

It follows that $\boldsymbol{B}$ is precisely the set of those $b \in \boldsymbol{C}$ for which $Q y_{b}=0$ where

$$
y_{b}=\frac{1}{T} U(T) \int_{0}^{T} U^{-1}(s) b(s) d s .
$$

This reduces to the well-known statements concerning the mean values of $b$ when the matrix $A(t)$ in (1) is a constant matrix of the form $\operatorname{diag}(0, C)$ and $C$ has no characteristic multipliers equal to 1.

From Theorem 2 and its proof we deduce the following result.

CoROLlary 2. Let $a \geqq 0$ and positive constants $k, r, \rho$ be chosen such that $\alpha a+\gamma \rho \leqq r,(k \gamma<1), k r<\rho$. If $f$ is lipschitzian in $K \times H$ for the constant $k /(2\|\tau\|)$ and $\|f(t, 0)\| \leqq(\rho-k r) /(2\|\tau\|)$ at any $t \in K$, there exists, for each $x_{0} \in H_{0}$, a unique continuously differentiable mapping $u_{x_{0}} \in \boldsymbol{V}$ with $\left\|u_{x_{0}}\right\| \leqq r$ for which $P u_{x_{0}}(0)=x_{0}$ and

$$
u_{x_{0}}^{\prime}(t)=A(t) u_{x_{0}}(t)+f\left(t, u_{x_{0}}(t)\right)-h_{u_{x_{0}}}(t)
$$


at every $t \in K$. Moreover, the mapping $x_{0} \rightarrow u_{x_{0}}$ is continuous in $H_{0}$.

A sequence $\left(v_{n}\right)$ of successive approximations to $u_{x_{0}}$ is easily determined by putting $v_{0}=0$ and defining each $v_{n}, n \geqq 1$, to be the unique solution of (3) with $b=f \circ g_{v_{n-1}}-h_{v_{n-1}}$ such that $v_{n} \in V$ and $P v_{n}(0)=x_{0}$.

The successive approximations which are used, for example, in [3] and [8] for proving results similar to Corollary 2 are chosen so as to belong to both $\boldsymbol{B}$ and $\boldsymbol{V}$. Though perhaps less convenient, such a choice is possible because (3) has for every $b \in \boldsymbol{B}$ a unique solution in $\boldsymbol{B} \cap \boldsymbol{V}$. This results at once, in our setting, from the following more general fact.

The projection $\tau(v)$ of any solution $v \in \boldsymbol{V}$ of (3) with $b \in \boldsymbol{B}$ is the solution of the homogeneous equation associated with (3) whose value at $t=T$ is

$$
x_{T}=Q\left[v(0)-\frac{1}{T} U(T) \int_{0}^{T} U^{-1}(s) b(s) s d s\right] .
$$

Conversely, each $x_{T} \in Y_{0}$ determines for every $b \in \boldsymbol{B}$ one and only one solution $v \in V$ of (3) for which $\tau(v)$ has the value $x_{T}$ at $t=T$. Hence $v \in V$ is uniquely determined by either $x_{0} \in X_{0}$ or $x_{T} \in Y_{0}$; in particular, $x_{T}=0$ implies $v \in \boldsymbol{B} \cap \boldsymbol{V}$.

\section{REFERENCES}

1. H. A. Antosiewicz, On the existence of periodic solutions of non-linear differential equations, Colloques Internat. du Centre National de la Recherche Scient. No. 148, 1964, 213-216 (1965).

2. L. Cesari, Asymptotic behavior and stability problems in ordinary differential equations, Springer-Verlag, 1959.

3. L. Cesari, Functional Analysis and periodic solutions of nonlinear differential equations, Contributions to Diff. Equations 1 (1963), 149-187.

4. R. Conti, Problèmes linéaires pour les équations différentielles ordinaires, Math. Nachrichten 23 (1961), 161-178.

5. R. Conti, Equazioni differenziali ordinarie quasilineari con condizioni lineari, Ann. Mat. Pura Appl. 57 (1962), 48-62.

6. R. Conti, On non-linear boundary value type problems, RIAS Tech. Rep. 64-12, 1964.

7. C. Corduneanu, Sur certain systèmes différentiels non-linéaries, An. Sti. Univ. "Al.

I. Cuza" 6 (1960), 257-260.

8. J. K. Hale, Oscillations in nonlinear systems, McGraw-Hill, 1963.

9. P. Hartman and N. Onuchic, On the asymptotic integration of ordinary differential equations, Pacific J. Math. 13 (1963), 1193-1207.

10. J. L. Massera, Sur l'existence de solutions bornées et périodiques des systèmes quasilinéaires d'équations différentielles, Ann. Mat. pura appl. 51 (1960), 95-105.

11. J. L. Massera and J. J. Schäffer, Linear differential equations and functional analysis, II, Ann. Math. 69 (1959), 88-104.

12. J. L. Massera and J. J. Schäffer, Linear differential equations and functional analysis, IV, Math. Ann. 139 (1960), 287-342. 



\section{PACIFIC JOURNAL OF MATHEMATICS}

\section{EDITORS}

\author{
H. SAMELSON \\ Stanford University \\ Stanford, California \\ R. M. BLUMENTHAL \\ University of Washington \\ Seattle, Washington 98105
}

\author{
*J. DUGUNDJI \\ University of Southern California \\ Los Angeles, California 90007
}

RICHARD ARENS

University of California Los Angeles, California 90024

\section{ASSOCIATE EDITORS}
E. F. BECKENBACH
B. H. NEUMANN
F. WOLF
K. YoSIDA

\section{SUPPORTING INSTITUTIONS}

\author{
UNIVERSITY OF BRITISH COLUMBIA \\ CALIFORNIA INSTITUTE OF TECHNOLOGY \\ UNIVERSITY OF CALIFORNIA \\ MONTANA STATE UNIVERSITY \\ UNIVERSITY OF NEVADA \\ NEW MEXICO STATE UNIVERSITY \\ OREGON STATE UNIVERSITY \\ UNIVERSITY OF OREGON \\ OSAKA UNIVERSITY \\ UNIVERSITY OF SOUTHERN CALIFORNIA
}

\author{
STANFORD UNIVERSITY \\ UNIVERSITY OF TOKYO \\ UNIVERSITY OF UTAH \\ WASHINGTON STATE UNIVERSITY \\ UNIVERSITY OF WASHINGTON \\ * * * * \\ AMERICAN MATHEMATICAL SOCIETY \\ CHEVRON RESEARCH CORPORATION \\ TRW SYSTEMS \\ NAVAL ORDNANCE TEST STATION
}

Mathematical papers intended for publication in the Pacific Journal of Mathematics should be typewritten (double spaced). The first paragraph or two must be capable of being used separately as a synopsis of the entire paper. It should not contain references to the bibliography. Manuscripts may be sent to any one of the four editors. All other communications to the editors should be addressed to the managing editor, Richard Arens at the University of California, Los Angeles, California 90024.

50 reprints per author of each article are furnished free of charge; additional copies may be obtained at cost in multiples of 50 .

The Pacific Journal of Mathematics is published monthly. Effective with Volume 16 the price per volume (3 numbers) is $\$ 8.00$; single issues, $\$ 3.00$. Special price for current issues to individual faculty members of supporting institutions and to individual members of the American Mathematical Society: $\$ 4.00$ per volume; single issues $\$ 1.50$. Back numbers are available.

Subscriptions, orders for back numbers, and changes of address should be sent to Pacific Journal of Mathematics, 103 Highland Boulevard, Berkeley 8, California.

Printed at Kokusai Bunken Insatsusha (International Academic Printing Co., Ltd.), No. 6, 2-chome, Fujimi-cho, Chiyoda-ku, Tokyo, Japan.

\section{PUBLISHED BY PACIFIC JOURNAL OF MATHEMATICS, A NON-PROFIT CORPORATION}

The Supporting Institutions listed above contribute to the cost of publication of this Journal, but they are not owners or publishers and have no responsibility for its content or policies.

* Paul A. White, Acting Editor until J. Dugundji returns. 


\section{Pacific Journal of Mathematics}

\section{Vol. 17, No. 2 \\ February, 1966}

Henry A. Antosiewicz, Boundary value problems for nonlinear ordinary

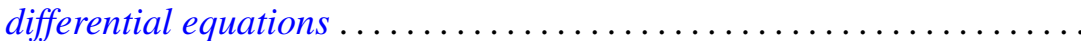

Bernard Werner Levinger and Richard Steven Varga, Minimal Gerschgorin sets. II ........................................

Paul Camion and Alan Jerome Hoffman, On the nonsingularity of complex matrices........................................ 211

J. Chidambaraswamy, Divisibility properties of certain factorials ........ 215

J. Chidambaraswamy, A problem complementary to a problem of Erdös .... 227

John Dauns, Chains of modules with completely reducible quotients ...... 235

Wallace E. Johnson, Existence of half-trajectories in prescribed regions and asymptotic orbital stability ............................ 243

Victor Klee, Paths on polyhedra. II . ........................ 249

Edwin Haena Mookini, Sufficient conditions for an optimal control problem in the calculus of variations ............................ 263

Zane Clinton Motteler, Existence theorems for certain quasi-linear elliptic equations........................................... 279

David Lewis Outcalt, Simple n-associative rings ............... 301

David Joseph Rodabaugh, Some new results on simple algebras ......... 311

Oscar S. Rothaus, Asymptotic properties of groups generation ........... 319

Ernest Edward Shult, Nilpotence of the commutator subgroup in groups admitting fixed point free operator groups .............

William Hall Sills, On absolutely continuous functions and the

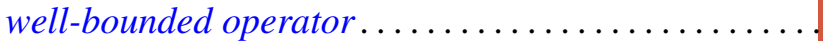

Joseph Gail Stampfli, Which weighted shifts are subnormal ..

Donald Reginald Traylor, Metrizability and completeness in normal Moore spaces 